\title{
LINEARLY INVARIANT FAMILIES OF HOLOMORPHIC FUNCTIONS IN THE UNIT POLYDISC
}

\author{
JANUSZ GODULA \\ Institute of Mathematics, Maria Curie-Sktodowska University \\ PL-20-031 Lublin, Poland \\ E-mail: godula@hektor.umcs.lublin.pl \\ VICTOR STARKOV \\ Department of Mathematics, University of Petrozavodsk \\ Petrozavodsk, Russia \\ E-mail: starkov@mainpgu.karelia.ru
}

\begin{abstract}
In this paper we extend the definition of the linearly invariant family and the definition of the universal linearly invariant family to higher dimensional case. We characterize these classes and give some of their properties. We also give a relationship of these families with the Bloch space.
\end{abstract}

1. Introduction. Ch. Pommerenke has introduced ([1]) the notion of a linearly invariant family $M$ as a class of functions $f$ holomorphic in the unit disc $\Delta=\{z: z \in$ $\mathbb{C},|z|<1\}$ such that

1) $f(0)=0, f^{\prime}(0)=1, f^{\prime}(z) \neq 0$ in $\Delta$,

2) for all $f \in M$ and $\theta \in \mathbb{R}, f\left(z e^{i \theta}\right) e^{-i \theta} \in M$,

$3)$ for all $f \in M$ and $a \in \Delta \quad f_{a}(z):=\frac{f\left(\frac{z+a}{1+\bar{a} z}\right)-f(a)}{f^{\prime}(a)\left(1-|a|^{2}\right)}=z+\cdots \in M$.

The number

$$
\operatorname{ord} f=\sup _{a \in \Delta} \frac{\left|f_{a}^{\prime \prime}(0)\right|}{2}
$$

was called, by Ch. Pommerenke ([1]), the order of a locally univalent function $f$, and the number

$$
\operatorname{ord} M=\sup _{f \in M} \operatorname{ord} f
$$

- the order of the family $M$. Moreover,

$$
\bigcup\{M: \operatorname{ord} M \leq \alpha\}:=U_{\alpha}
$$

1991 Mathematics Subject Classification: Primary 32A10; Secondary 30C55.

The paper is in final form and no version of it will be published elsewhere. 
was called the universal linearly invariant family.

Linearly invariant families play an important part in the theory of conformal mappings. Furthermore an interest in the families $U_{\alpha}$ grows, because of their relationship with the Bloch class ([2]).

The main goal of this paper is to extend the definition of the linearly invariant families onto the case of functions defined on the unit polydisc $\Delta^{m} \subset \mathbb{C}^{m}, m \geq 1$, and establish several properties

Let $\mathbb{T}=\{z: z \in \mathbb{C},|z|=1\}$ and $\mathbb{T}^{m}$ be the unit torus. We will consider the class $H\left(\Delta^{m}\right)$ of all functions $f: \Delta^{m} \longrightarrow \mathbb{C}$ holomorphic in $\Delta^{m}$. The gradient of a holomorphic function $f$ we denote by $\nabla f$; that is $\nabla f=\left(\frac{\partial f}{\partial z_{1}}, \cdots, \frac{\partial f}{\partial z_{m}}\right)$. For $z=\left(z_{1}, \cdots, z_{m}\right) \in \mathbb{C}^{m}$ we define the norm

$$
\|z\|=\max _{1 \leq j \leq m}\left|z_{j}\right| .
$$

Let $\mathbf{O}=(0, \cdots, 0) \in \mathbb{C}^{m}$. Recall that to every $a \in \Delta$ corresponds an automorphism $\phi_{a}$ of $\Delta: \phi_{a}(z)=(a+z) /(1+\bar{a} z), z \subset \Delta$. The same can be done in the polydisc $\Delta^{m}$. For $a=\left(a_{1}, \cdots, a_{m}\right) \in \Delta^{m}$ the Möbius mapping $\phi_{a}$ of $\Delta^{m}$ onto $\Delta^{m}$ we define by the formula

$$
\phi_{a}(z)=\left(\phi_{1}\left(z_{1}\right), \cdots, \phi_{m}\left(z_{m}\right)\right) \text {, }
$$

where

$$
\phi_{j}\left(z_{j}\right)=\frac{z_{j}+a_{j}}{1+\overline{a_{j}} z_{j}}, \quad j=1, \cdots, m .
$$

Now, we are ready to introduce the linearly (Möbius) invariant family.

Definition 1.1. Let $l=1, \cdots, m$ be fixed. The $l$-Möbius invariant family $M_{l}$ is the class of all functions $f, f \in H\left(\Delta^{m}\right)$, such that

1) $f(\mathbf{O})=0, \quad \frac{\partial f}{\partial z_{l}}(\mathbf{O})=1, \quad \frac{\partial f}{\partial z_{l}}(z) \neq 0, \quad$ for $z \in \Delta^{m}$,

2) for all $f \in M_{l}$ and $\theta=\left(\theta_{1}, \cdots, \theta_{m}\right) \in \mathbb{R}^{m}, f\left(z e^{i \theta}\right) e^{-i \theta_{l}} \in M$, where $z e^{i \theta}=$ $\left(z_{1} e^{i \theta_{1}}, \cdots, z_{m} e^{i \theta_{m}}\right)$.

3) for all $f \in M_{l}$ and $a=\left(a_{1}, \cdots, a_{m}\right) \in \Delta^{m}$,

$$
f_{a}(z):=\frac{f\left(\phi_{a}(z)\right)-f\left(\phi_{a}(\mathbf{O})\right)}{\frac{\partial f}{\partial z_{l}}(a)\left(1-\left|a_{l}\right|^{2}\right)} \in M_{l} .
$$

EXAMPLES:

(i) $K_{l}$ - the class of functions $f \in H\left(\Delta^{m}\right)$ satisfying 1$)$ of the above Definition and such that $f\left(\Delta^{m}\right)$ is a convex domain.

(ii) $S_{l}^{*}$ - the class of functions $f \in H\left(\Delta^{m}\right)$ satisfying 1) of the above Definition and such that there exists a point $w_{f} \in f\left(\Delta^{m}\right)$, such that the domain $f\left(\Delta^{m}\right)$ is starlike with respect to $w_{f}$.

(iii) $S_{l}^{k}$, where $k=1, \cdots, m$ is fixed, - the class of all functions $f_{k} \in H\left(\Delta^{m}\right)$ satisfying 1) of the above Definition such that $F(z)=\left(f_{1}(z), \cdots, f_{m}(z)\right)$ is a univalent mapping of $\Delta^{m}$ into $\mathbb{C}^{m}$.

The following definition extends the Pommerenke's conception of the order of a function, ([1]). 
Definition 1.2. Let $f$ satisfy the conditions 1$)$ of Definition 1.1 and let $\frac{\partial f_{a}}{\partial z_{l}}(z)=$ $1+c_{1}(f) z_{1}+\cdots+c_{m}(f) z_{m}+o(\|z\|)$. The $l$-order of the function $f$ is defined as follows:

$$
\operatorname{ord}_{l} f=\sup _{a \in \Delta^{m}} \frac{1}{2}\left\|\nabla \frac{\partial f_{a}}{\partial z_{l}}(\mathbf{O})\right\|=\frac{1}{2} \sup _{a \in \Delta^{m}}\left\|\left(c_{1}\left(f_{a}\right), \cdots, c_{m}\left(f_{a}\right)\right)\right\| .
$$

THEOREM 1.1 If $f \in M_{l}, a \in \Delta^{m}$, then

$$
\begin{gathered}
\operatorname{ord}_{l} f=\max _{1 \leq k \leq m} \sup _{z \in \Delta^{m}}\left|\frac{\frac{\partial^{2} f}{\partial z_{l} \partial z_{k}}}{\frac{\partial f}{\partial z_{l}}(z)} \frac{1-\left|z_{k}\right|^{2}}{2}-\overline{z_{k}} \delta_{k}^{l}\right|= \\
\sup _{a \in \Delta^{m}}\left\|\frac{1}{2} \nabla \log \left(\frac{\partial f}{\partial z_{l}} \circ \phi_{a}\right)(\mathbf{O})-\left(0, \cdots, 0, \overline{a_{l}}, 0, \cdots, 0\right)\right\|,
\end{gathered}
$$

where

$$
\delta_{k}^{l}= \begin{cases}1, & \text { for } k=l \\ 0, & \text { for } k \neq l\end{cases}
$$

Proof. Let us observe that

Then

$$
\frac{\partial f_{a}}{\partial z_{l}}(z)=\frac{\left(\frac{\partial f}{\partial z_{l}} \circ \phi_{a}\right)(z)}{\frac{\partial f}{\partial z_{l}}(a)\left(1+\overline{a_{l}} z_{l}\right)^{2}}
$$

$$
\left\|\nabla \frac{\partial f_{a}}{\partial z_{l}}(\mathbf{O})\right\|=\max _{1 \leq k \leq m}\left|\frac{\frac{\partial^{2} f}{\partial z_{l} \partial z_{k}}(a)}{\frac{\partial f}{\partial z_{l}}(a)}\left(1-\left|a_{k}\right|^{2}\right)-2 \overline{a_{l}} \delta_{k}^{l}\right| .
$$

The above gives the result.

Now, we introduce the order of a family $M_{l}$.

Definition 1.3. The $l$-order of a $l$-Möbius invariant family $M_{l}$ is defined as

$$
\operatorname{ord}_{l} M_{l}=\sup _{f \in M_{l}} \operatorname{ord}_{l} f \text {. }
$$

EXAMPLES:

1) $M_{l}=\left\{f(z)=\Phi\left(z_{l}\right): \Phi \in U_{\alpha}\right\}$ is the $l$-Möbius invariant family of the $l$-order $\alpha$.

2) Let $k \neq l$ and let $\Phi_{k}\left(z_{k}\right)$ be functions holomorphic in $\Delta$ such that $\Phi_{k}(0)=0$. Then

$$
M_{l}=\left\{f(z)=\sum_{k=1}^{\infty} \lambda_{k} \Phi_{k}\left(z_{k}\right): \Phi_{l} \in U_{\alpha}, \lambda_{k} \in \mathbb{C}, \lambda_{l}=1\right\}
$$

is the $l$-Möbius invariant family of the $l$-order $\alpha$.

3) Let

$$
\Psi(z)=\frac{1}{2 \alpha}\left[\prod_{k=1}^{m}\left(\frac{1+z_{k}}{1-z_{k}}\right)^{\alpha}-1\right] .
$$

Then for all $l=1, \cdots, m$ the class

$$
\left\{\Psi_{a}\left(z e^{i \theta}\right) e^{-i \theta_{l}}: \quad a \in \Delta^{m}, \quad \theta \in \mathbb{R}^{m}\right\}
$$

is the $l$-Möbius invariant family of the $l$-order $\alpha$.

2. Universal linearly (Möbius) invariant family. In the next definition we introduce a universal linearly (Möbius) invariant family. 
Definition 2.1. The universal $l$-Möbius invariant family $U_{\alpha}^{l}$ of the $l$-order $\alpha$ is defined as the union of all families $M_{l}$ such that $\operatorname{ord}_{l} M_{l} \leq \alpha$; that is

$$
U_{\alpha}^{l}=\cup\left\{M_{l}: \operatorname{ord}_{l} M_{l} \leq \alpha\right\} .
$$

TheOREM 2.1. For any $f \in U_{\alpha}^{l}$ and all $z \in \Delta^{m}$ we have

$$
\begin{gathered}
\left|\log \left(\left(1-\left|z_{l}\right|^{2}\right) \frac{\partial f}{\partial z_{l}}(z)\right)\right| \leq \alpha \log \prod_{k=1}^{m} \frac{1+\left|z_{k}\right|}{1-\left|z_{k}\right|}, \\
\frac{1}{1-\left|z_{l}\right|^{2}} \prod_{k=1}^{m}\left(\frac{1-\left|z_{k}\right|}{1+\left|z_{k}\right|}\right)^{\alpha} \leq\left|\frac{\partial f}{\partial z_{l}}(z)\right| \leq \frac{1}{1-\left|z_{l}\right|^{2}} \prod_{k=1}^{m}\left(\frac{1+\left|z_{k}\right|}{1-\left|z_{k}\right|}\right)^{\alpha} .
\end{gathered}
$$

The above inequalities are rendered by the functions

$$
\Psi(z)=\frac{1}{2 \alpha}\left[\prod_{k=1}^{m}\left(\frac{1+z_{k}}{1-z_{k}}\right)^{\alpha}-1\right],
$$

for $\alpha \geq 1$ and real $z_{k}$.

Proof. From Theorem 1.1 we have

$$
\left|\frac{\frac{\partial^{2} f}{\partial z_{l}^{2}}(z)}{\frac{\partial f}{\partial z_{l}}(z)} \frac{1-\left|z_{l}\right|^{2}}{2}-\overline{z_{l}}\right| \leq \alpha .
$$

Thus for $z_{l}=r_{l} e^{i \theta_{l}}$ (if $z_{l} \neq 0$ ) we get

$$
\left|\frac{\partial}{\partial r_{l}}\left[\log \left(\frac{\partial f}{\partial z_{l}}(z)\left(1-r_{l}^{2}\right)\right)\right]\right|=\left|\frac{\frac{\partial^{2} f}{\partial z_{l}^{2}}(z)}{\frac{\partial f}{\partial z_{l}}(z)} e^{i \theta_{l}}-\frac{2 r_{l}}{1-r_{l}^{2}}\right| \leq \frac{2 \alpha}{1-r_{l}^{2}} .
$$

Now, let

$$
z_{l}=\left(z_{1}, \cdots, z_{l-1}, 0, z_{l+1}, \cdots, z_{m}\right) .
$$

Then we obtain

$$
\begin{aligned}
\left|\log \left(\frac{\partial f}{\partial z_{l}}(z)\left(1-r_{l}^{2}\right)\right)-\log \frac{\partial f}{\partial z_{l}}\left(z^{l}\right)\right| & =\left|\int_{0}^{r} \frac{\partial}{\partial r_{l}}\left[\log \left(\frac{\partial f}{\partial z_{l}}(z)\left(1-r_{l}^{2}\right)\right)\right] d r_{l}\right| \leq \\
\int_{0}^{r_{l}} \frac{2 \alpha}{1-r_{l}^{2}} d r_{l} & =\alpha \log \frac{1+r_{l}}{1-r_{l}}
\end{aligned}
$$

By Theorem 1.1 we have

$$
\left|\frac{\frac{\partial^{2} f}{\partial z_{l} \partial z_{k}}(z)}{\frac{\partial f}{\partial z_{l}}(z)}\right| \leq \frac{2 \alpha}{1-r_{k}^{2}}
$$

for all $k \neq l$ and $z \in \Delta^{m}$.

Let $z^{l, k}$ be a point in $\Delta^{m}$ for which $z_{l}=z_{k}=0$. Then

$$
\begin{aligned}
\left|\log \frac{\partial f}{\partial z_{l}}\left(z^{l}\right)-\log \frac{\partial f}{\partial z_{l}}\left(z^{l, k}\right)\right| & =\left|\int_{0}^{r_{k}} \frac{\frac{\partial^{2} f}{\partial z_{l} \partial z_{k}}\left(z^{l}\right)}{\frac{\partial f}{\partial z_{l}}\left(z^{l}\right)} e^{i \theta_{k}} d r_{k}\right| \leq \\
\int_{0}^{r_{k}} \frac{2 \alpha}{1-r_{k}^{2}} d r_{k} & =\alpha \log \frac{1+r_{k}}{1-r_{k}} .
\end{aligned}
$$


Now, if $l \neq p \neq k, l \neq k$, then analogously to (2.4) we get

$$
\left|\log \frac{\partial f}{\partial z_{l}}\left(z^{l, k}\right)-\log \frac{\partial f}{\partial z_{l}}\left(z^{l, k, p}\right)\right| \leq \alpha \log \frac{1+r_{p}}{1-r_{p}},
$$

where a point $z^{l, k, p} \in \Delta^{m}$, and $z_{l}=z_{k}=z_{p}=0$. Using the above scheme we obtain, in the end, an estimation of the type (2.5) of the expression

$$
\left|\log \frac{\partial f}{\partial z_{l}}\left(z^{*}\right)-\log \frac{\partial f}{\partial z_{l}}(\mathbf{O})\right|
$$

where a point $z^{*} \in \Delta^{m}$ and it has only one component different from zero. Summing $(2.3),(2.4),(2.5), \ldots$ we obtain (2.1). If we put the function $\Psi$ in $(2.1)$, with $z_{k}=\left|z_{k}\right|$, for all $k=1, \cdots, m$ then we have the equality in $(2.1)$.

For the proof of (2.2), let us observe that from (2.1) we obtain

$$
\begin{gathered}
\alpha \log \prod_{k=1}^{m} \frac{1-\left|z_{k}\right|}{1+\left|z_{k}\right|}-\log \left(1-\left|z_{l}\right|^{2}\right) \leq \Re\left[\log \frac{\partial f}{\partial z_{l}}(z)-\log \frac{\partial f}{\partial z_{l}}(\mathbf{O})\right] \leq \\
\alpha \log \prod_{k=1}^{m} \frac{1+\left|z_{k}\right|}{1-\left|z_{k}\right|}-\log \left(1-\left|z_{l}\right|^{2}\right) .
\end{gathered}
$$

For the function $\Psi$, with $z_{k}= \pm\left|z_{k}\right|$ for all $k=1, \cdots, m$, we have the equality in (2.2).

Remark. For $m=1$ the above Theorem gives the well known result for the class $U_{\alpha},([1])$.

Corollary. In the Definition of $U_{\alpha}^{l}$ we have $\alpha \geq 1$, because $U_{\alpha}^{l}=\emptyset$ for $\alpha<1$.

Indeed, if we suppose that $\alpha<1$, then from (2.2) it follows that $\lim _{\left|z_{l}\right| \rightarrow 1^{-}}\left|\frac{\partial f}{\partial z_{l}}(z)\right|=$ $\infty$ for fixed rest components of $z=\left(z_{1}, \cdots, z_{l}, \cdots, z_{m}\right)$. The holomorphic, with respect to $z_{l}$, function $\frac{\partial f}{\partial z_{l}}$ is not equal zero. Thus $\min _{|z|<r}\left|\frac{\partial f}{\partial z_{l}}(z)\right|$ is attained on $\left\{\left|z_{l}\right|=r\right\}$ and this minimum tends to $\infty$, if $r \rightarrow 1^{-}$. The above contradicts $\frac{\partial f}{\partial z_{l}}(\mathbf{O})=1$.

THEOREM 2.2. The family $U_{\alpha}^{l}$ is the set of all functions holomorphic in $\Delta^{m}$ and satisfying the conditions 1), 2), 3) of Definition 1.1 and the condition (2.2) in a neighbourhood of $\mathbf{O}$.

Proof. Let $F$ be a family of functions satisfying the conditions mentioned in our theorem. It is enough to show that $F \subset U_{\alpha}(l)$. Let $f \in F$. Thus, by 2), for all $z$ from a neighourhood of $\mathbf{O}$ we have

$$
\alpha \log \prod_{k=1}^{m} \frac{1-r_{k}}{1+r_{k}}-\log \left(1-r_{l}^{2}\right) \leq \Re \log \frac{\partial f}{\partial z_{l}}(z) \leq \alpha \log \prod_{k=1}^{m} \frac{1+r_{k}}{1-r_{k}}-\log \left(1-r_{l}^{2}\right),
$$

where $z_{k}=r_{k} e^{i \theta_{k}}$.

The above inequalities are true for a function $f_{a}$, for every $a \in \Delta^{m}$. For $z=\mathbf{O}$ both left and right expression of (2.6) are 0. Thus, after the differentiation, with respect to $r_{k}$, $k=1, \cdots, m$, of $(2.6)$ in the point $\mathbf{O}$ we get (if $k \neq l$ )

$$
-2 \alpha \leq \Re \frac{\frac{\partial^{2} f_{a}}{\partial z_{l} \partial z_{k}}(\mathbf{O})}{\frac{\partial f_{a}}{\partial z_{l}}(\mathbf{O})} \leq 2 \alpha
$$


which is equivalent to

$$
\left|\frac{\frac{\partial^{2} f}{\partial z_{l} \partial z_{k}}(a)}{\frac{\partial f}{\partial z_{l}}(a)}\left(1-\left|a_{k}\right|^{2}\right)\right| \leq 2 \alpha
$$

Moreover, if $k=l$ we get

$$
-2 \alpha \leq \Re\left[\frac{\frac{\partial^{2} f}{\partial z_{l}^{2}}\left(1-\left|a_{l}\right|^{2}\right)-2 \overline{a_{l}} \frac{\partial f}{\partial z_{l}}(a)}{\frac{\partial f}{\partial z_{l}}(a)} e^{i \gamma_{l}}\right] \leq 2 \alpha,
$$

for all $\gamma_{l} \in \mathbb{R}$; which is equivalent to

$$
\left|\frac{\frac{\partial^{2} f}{\partial z_{l}^{2}}(a)}{\frac{\partial f}{\partial z_{l}}(a)}\left(1-\left|a_{l}\right|^{2}\right)-2 \overline{a_{l}}\right| \leq 2 \alpha .
$$

From (2.7) and (2.8) it follows that $\operatorname{ord}_{l} f \leq \alpha$. Thus $f \in U_{\alpha}^{l}$.

Remark. For $m=1$ we get known result in the class $U_{\alpha},([3])$.

Now, let for $x \in[0,1), q \in[-1,1]$

$$
\begin{gathered}
\Xi(x, q)=\int_{0}^{x} \frac{\sqrt{1-q^{2} t^{2}}}{1-t^{2}} d t=\frac{1}{2} \sqrt{1-q^{2}} \log \frac{\sqrt{1-q^{2} x^{2}}+x \sqrt{1-q^{2}}}{\sqrt{1-q^{2} x^{2}}-x \sqrt{1-q^{2}}}+q \arcsin x \leq \\
\frac{1}{2} \sqrt{1-q^{2}} \log \frac{1+x}{1-x}+\arcsin x .
\end{gathered}
$$

Observe, that the function $\alpha \Xi\left(|z|, \frac{\sin \lambda}{\alpha}\right)$ is increasing with respect to $\alpha$.

In the paper [1] Ch. Pommerenke has obtained an estimate of $\left|\Re\left\{e^{-i \lambda} \log f^{\prime}(z)\right\}\right|$ in the class $U_{\alpha}$. Now, we give a similar result for the class $U_{\alpha}^{l}$.

THEOREM 2.3. For all $f \in U_{\alpha}^{l}$ and all real $\lambda$

$$
\left|\Re\left\{e^{-i \lambda} \log \frac{\partial f}{\partial z_{l}}(z)\left(1-\left|z_{l}\right|^{2}\right)\right\}\right| \leq \alpha\left(\log \prod_{k \neq l} \frac{1+\left|z_{k}\right|}{1-\left|z_{k}\right|}+2 \Xi\left(\left|z_{l}\right|, \frac{|\sin \lambda|}{\alpha}\right)\right) .
$$

Proof. Let us denote

$$
u\left(r_{1}, \cdots, r_{m}\right)=\max _{\left|z_{k}\right| \leq r_{k}} \Re\left\{e^{-i \lambda} \log \frac{\partial f}{\partial z_{l}}\left(z_{1}, \cdots, z_{m}\right)\right\} .
$$

By the maximum principle for harmonic functions

$$
u\left(r_{1}, \cdots, r_{m}\right)=\Re\left\{e^{-i \lambda} \log \frac{\partial f}{\partial z_{l}}\left(r_{1} e^{i \theta_{1}^{(0)}\left(r_{1}, \cdots, r_{m}\right)}, \cdots, r_{m} e^{i \theta_{m}^{(0)}\left(r_{1}, \cdots, r_{m}\right)}\right)\right\} .
$$

Then

$$
\begin{gathered}
-\left.\frac{\partial}{\partial \theta_{k}}\left(\Re\left\{e^{-i \lambda} \log \frac{\partial f}{\partial z_{l}}\left(r_{1} e^{i \theta_{1}}, \cdots, r_{m} e^{i \theta_{m}}\right)\right\}\right)\right|_{\theta=\theta^{(0)}}= \\
\Im\left\{e^{-i \lambda} \frac{\frac{\partial^{2} f}{\partial z_{l} \partial z_{k}}}{\frac{\partial f}{\partial z_{l}}}\left(r_{1} e^{i \theta_{1}^{(0)}}, \cdots, r_{m} e^{i \theta_{m}^{(0)}}\right) e^{i \theta_{k}^{(0)}} r_{k}\right\}=0
\end{gathered}
$$

where $\theta^{(0)}=\theta^{(0)}\left(r_{1}, \cdots, r_{m}\right)=\left(\theta_{1}^{(0)}\left(r_{1}, \cdots, r_{m}\right), \cdots, \theta_{m}^{(0)}\left(r_{1}, \cdots, r_{m}\right)\right)$. The function $u\left(r_{1}, \cdots, r_{m}\right)$ increases with respect to every variable $r_{k} \in[0,1)$. Thus, by the Lebesgue 
theorem everywhere on $[0,1)$ there exists finite derivative $\frac{\partial u}{\partial r_{k}}\left(r_{1}, \cdots, r_{k-1}, t r_{k}, \cdots, r_{m}\right)$. Then

$$
\begin{gathered}
\Re\left\{e^{-i \lambda} \log \frac{\partial f}{\partial z_{l}}\left(r_{1} e^{i \theta_{1}^{(0)}}, \cdots, r_{k-1} e^{i \theta_{1}^{(0)}}, t e^{i \theta_{k}^{(0)}}, r_{k+1} e^{i \theta_{k+1}^{(0)}}, \cdots, r_{m} e^{i \theta_{m}^{(0)}}\right)\right\} \leq \\
u\left(r_{1}, \cdots, r_{k-1}, t, r_{k+1}, \cdots, r_{m}\right),
\end{gathered}
$$

with equality for $t=r_{k}$. From the above it follows,that for almost all $r_{k}$ :

$$
\begin{gathered}
\Re\left\{e^{-i \lambda} \frac{\frac{\partial^{2} f}{\partial z_{l} \partial z_{k}}}{\frac{\partial f}{\partial z_{l}}}\left(r_{1} e^{i \theta_{1}^{(0)}}, \cdots, r_{k} e^{i \theta_{k}^{(0)}}, \cdots, r_{m} e^{i \theta_{m}^{(0)}}\right) e^{i \theta_{k}^{(0)}}\right\}= \\
\frac{\partial u}{\partial r_{k}}\left(r_{1}, \cdots, r_{k}, \cdots, r_{m}\right) .
\end{gathered}
$$

By (2.9) we get

$$
\frac{\partial u}{\partial r_{k}}\left(r_{1}, \cdots, r_{k}, \cdots, r_{m}\right)=e^{-i \lambda} \frac{\frac{\partial^{2} f}{\partial z_{l} \partial z_{k}}}{\frac{\partial f}{\partial z_{l}}}\left(r_{1} e^{i \theta_{1}^{(0)}}, \cdots, r_{k} e^{i \theta_{k}^{(0)}}, \cdots, r_{m} e^{i \theta_{m}^{(0)}}\right) e^{i \theta_{k}^{(0)}} .
$$

From the above and from Theorem 1.1 it follows that for almost all $r_{k}$ (the rest of variables of the function $u$ are fixed) we have

$$
\begin{gathered}
\left|\frac{\partial u}{\partial r_{k}}\left(r_{1}, \cdots, r_{k}, \cdots, r_{m}\right) \frac{1-r_{k}^{2}}{2}-r_{k} \delta_{k}^{l} e^{-i \lambda}\right| \leq \alpha \\
\left(\frac{\partial u}{\partial r_{k}} \frac{1-r_{k}^{2}}{2}-r_{k} \delta_{k}^{l} \cos \lambda\right)^{2}+r_{k}^{2} \delta_{k}^{l} \sin ^{2} \lambda \leq \alpha^{2},
\end{gathered}
$$

and

$$
\left|\frac{\partial u}{\partial r_{k}}-2 \delta_{k}^{l} \frac{r_{k}}{1-r_{k}^{2}} \cos \lambda\right| \leq 2 \frac{\sqrt{\alpha^{2}-r_{k}^{2} \delta_{k}^{l} \sin ^{2} \lambda}}{1-r_{k}^{2}}
$$

For $k=l$ we have

$$
\left|\frac{\partial}{\partial r_{l}}\left[u+\cos \lambda \log \left(1-r_{l}^{2}\right)\right]\right| \leq 2 \frac{\sqrt{\alpha^{2}-r_{l}^{2} \sin ^{2} \lambda}}{1-r_{l}^{2}} .
$$

Integrating, we obtain

$$
\begin{gathered}
\left|u\left(r_{1}, \cdots, r_{l}, \cdots, r_{m}\right)+\cos \lambda \log \left(1-r_{l}^{2}\right)-u\left(r_{1}, \cdots, r_{l-1}, 0, r_{l+1}, \cdots, r_{m}\right)\right| \leq \\
2 \alpha \Xi\left(r_{l}, \frac{|\sin \lambda|}{\alpha}\right) ;
\end{gathered}
$$

and for $k \neq l$ :

$$
\begin{gathered}
\left|u\left(r_{1}, \cdots, r_{l-1}, 0, r_{l+1}, \cdots, r_{m}\right)-u\left(r_{1}, \cdots, r_{k-1}, 0, r_{k+1}, \cdots, r_{l-1}, 0, r_{l+1}, \cdots, r_{m}\right)\right| \leq \\
\int_{0}^{r_{k}}\left|\frac{\partial u}{\partial r_{k}}\left(r_{1}, \cdots, r_{k-1}, s, r_{k+1}, \cdots, r_{l-1}, 0, r_{l+1}, \cdots, r_{m}\right)\right| d s \leq 2 \int_{0}^{r_{k}} \frac{\alpha}{1-s^{2}} d s= \\
\alpha \log \frac{1+r_{k}}{1-r_{k}} .
\end{gathered}
$$


Having (m-1) similar inequalities with $k \neq l$ and summing them we obtain

$$
\left|u\left(r_{1}, \cdots, r_{m}\right)+\cos \lambda \log \left(1-r_{l}^{2}\right)-u(\mathbf{O})\right| \leq \alpha \sum_{k \neq l} \log \frac{1+r_{k}}{1-r_{k}}+2 \alpha \Xi\left(r_{l}, \frac{|\sin \lambda|}{\alpha}\right) .
$$

Thus we get the Theorem.

For $\lambda=2 \pi n, n$ an integer, we have Theorem 2.1, and for $e^{i \lambda}=i$ we get

Corollary.

$$
\begin{gathered}
\left|\arg \frac{\partial f}{\partial z_{l}}(z)\right| \leq \alpha\left(\log \prod_{k \neq l} \frac{1+\left|z_{k}\right|}{1-\left|z_{k}\right|}+2 \Xi\left(\left|z_{l}\right|, \frac{1}{\alpha}\right)\right) \leq \\
\alpha \log \prod_{k \neq l} \frac{1+\left|z_{k}\right|}{1-\left|z_{k}\right|}+\sqrt{\alpha^{2}-1} \log \frac{1+\left|z_{l}\right|}{1-\left|z_{l}\right|}+2 \arcsin \left|z_{l}\right| .
\end{gathered}
$$

For the proof see [1]. Here $\arg \frac{\partial f}{\partial z_{l}}(\mathbf{O})=0$ and it is continuous with respect to $z$. For $m=1$ we get Pommerenke's result for $U_{\alpha}$.

Remark. The above estimation is not rough.

To support this we give the following example of function.

$$
\begin{gathered}
\Psi_{0}(z)=\frac{1}{2 i \sqrt{\alpha^{2}-1}}\left[\prod_{k \neq l}\left(\frac{1+z_{k}}{1-z_{k}}\right)^{i \alpha}\left(\frac{1+z_{l}}{1-z_{l}}\right)^{i \sqrt{\alpha^{2}-1}}-1\right], \alpha>1 \\
\arg \Psi_{0}\left(r_{1}, \ldots, r_{m}\right)=\alpha \log \prod_{k \neq l} \frac{1+r_{k}}{1-r_{k}}+\sqrt{\alpha^{2}-1} \log \frac{1+r_{l}}{1-r_{l}} .
\end{gathered}
$$

Indeed

$$
\sup _{z \in \Delta^{m}}\left|\frac{\frac{\partial^{2} \Psi_{0}}{\partial z_{l}^{2}}}{\frac{\partial \Psi_{0}}{\partial z_{l}}} \frac{1-\left|z_{l}\right|^{2}}{2}-\bar{z}_{l}\right|=\sup _{\left|z_{l}\right|<1}\left|\frac{1-\left|z_{l}\right|^{2}}{1-z_{l}^{2}}\left(i \sqrt{\alpha^{2}-1}-1+2 z_{l}\right)-\bar{z}_{l}\right|=\alpha
$$

(see:[1], page 128). For all $k \neq l$

$$
\sup _{z \in \Delta^{m}}\left|\frac{\frac{\partial^{2} \Psi_{0}}{\partial z_{l} \partial z_{k}}}{\frac{\partial \Psi_{0}}{\partial z_{l}}} \frac{1-\left|z_{k}\right|^{2}}{2}\right|=\sup _{\left|z_{k}\right|<1}\left|\frac{\alpha i}{1-z_{k}^{2}}\left(1-\left|z_{k}\right|^{2}\right)\right|=\alpha
$$

By the Theorem 1.1 ord $l_{l} \Psi_{0}=\alpha$ and the equality (3.11) is fulfilled.

3. Bloch class. Now, we introduce the Bloch class of holomorphic functions.

Definition 3.1. A holomorphic function $g: \Delta^{m} \longrightarrow \mathbb{C}$ is called a Bloch function if

$$
\|g\|_{B}:=|g(\mathbf{O})|+\max _{k=1, \cdots, m} \sup _{z \in \Delta^{m}}\left|\frac{\partial g}{\partial z_{k}}\left(1-\left|z_{k}\right|^{2}\right)\right|<\infty .
$$

The set of all Bloch functions we will denote by $B:=B\left(\Delta^{m}\right)$.

The following result give a condition which is equivalent to the definition of the Bloch function. For $m=1$ the result was given by the authors in [2]. 
THEOREM 3.1. Let $l=1, \cdots, m$ be fixed. Then the following conditions are equivalent

(i) $g \in B$,

(ii) There exists $f \in \bigcup_{\alpha<\infty} U_{\alpha}^{l}$ such that

$$
g(z)-g(\mathbf{O})=\log \frac{\partial f}{\partial z_{l}}(z),
$$

where $\alpha=\operatorname{ord}_{l} f$. Moreover

$$
2(\alpha-1) \leq\|g(z)-g(\mathbf{O})\|_{B} \leq 2(\alpha+1) .
$$

Proof. $(i) \Rightarrow(i i)$ Let $g \in B$ and let $F$ be a function such that

$$
\frac{\partial F}{\partial z_{l}}(z)=\exp (g(z)-g(\mathbf{O})) .
$$

Now, let us consider a function $f$ defined by the formula

$$
f(z)=F(z)-F(\mathbf{O}) .
$$

One can see, that $f$ satisfies 1$)$ in Definition 1.1. Moreover

$$
\log \frac{\partial f}{\partial z_{l}}(z)=\log \frac{\partial F}{\partial z_{l}}(z)=g(z)-g(\mathbf{O}) .
$$

Since

$$
\alpha=\operatorname{ord}_{l} f=\max _{k=1, \cdots, m} \sup _{z \in \Delta^{m}}\left|\frac{\partial g}{\partial z_{k}}(z) \frac{1-\left|z_{k}\right|^{2}}{2}-\overline{z_{k}} \delta_{k}^{l}\right|
$$

we have

$$
\frac{1}{2}\|g(z)-g(\mathbf{O})\|_{B}-1 \leq \alpha \leq \frac{1}{2}\|g(z)-g(\mathbf{O})\|_{B}+1
$$

and $f \in U_{\alpha}^{l}$.

$($ ii $) \Rightarrow(i)$ Let $f \in \bigcup_{\alpha<\infty} U_{\alpha}^{l}$ and $\operatorname{ord}_{l} f=\alpha$. Let

$$
g(z)=\log \frac{\partial f}{\partial z_{l}}(z) .
$$

We have $g(\mathbf{O})=0$. From Theorem 2.1 it follows that for every $k=1, \cdots, m$

$$
\sup _{z \in \Delta^{m}}\left(\left|\frac{\partial g}{\partial z_{k}}(z)\right|\left(1-\left|z_{k}\right|^{2}\right)\right) \leq 2 \alpha+2 \delta_{l}^{k} \leq 2(\alpha+1) .
$$

Thus $g \in B$ and

$$
2(\alpha-1)\|g\|_{B} \leq \max _{k=1, \cdots, m} \sup _{z \in \Delta^{m}}\left(\left|\frac{\partial g}{\partial z_{k}}(z)\right|\left(1-\left|z_{k}\right|^{2}\right)\right) \leq 2(\alpha+1) .
$$

Now, let us give some properties of Bloch functions in terms of the order.

TheOrem 3.2. Let $g$ be a holomorphic function in $\Delta^{m}$. Then $g \in B$ if and only if there exists a positive constant $C_{g}$ such that for all $z \in \Delta^{m}$,

$$
\sup _{a \in \Delta^{m}}\left|g\left(\phi_{a}(z)\right)-g(a)-2 \log \left(1+\overline{a_{l}} z_{l}\right)+\log \left(1-\left|z_{l}\right|^{2}\right)\right| \leq C_{g} \log \prod_{k=1}^{m} \frac{1+\left|z_{k}\right|}{1-\left|z_{k}\right|} .
$$

The best value of the constant $C_{g}$ is equal ord $\int_{0}^{z_{l}} \exp g\left(z_{1}, \cdots, z_{l-1}, s, \cdots, z_{m}\right) d s$.

Proof. Let us suppose that $g(\mathbf{O})=0$. 
$1^{0}$. Let $g \in B$. Then there exists a function $f \in U_{\alpha}^{l}$ such that $g(z)=\log \frac{\partial f}{\partial z_{l}}(z)$. Since

we get

$$
f_{a}(z):=\frac{f\left(\phi_{a}(z)\right)-f\left(\phi_{a}(\mathbf{O})\right)}{\frac{\partial f}{\partial z_{l}}(a)\left(1-\left|a_{l}\right|^{2}\right)}
$$

$$
\frac{\partial f_{a}}{\partial z_{l}}(z)=\frac{\frac{\partial f}{\partial z_{l}}\left(\phi_{a}(z)\right)}{\frac{\partial f}{\partial z_{l}}(a)\left(1+\overline{a_{l}} z_{l}\right)^{2}}
$$

By $(2.1)$

$$
\log \left(\left(1-\left|z_{l}\right|^{2}\right) \frac{\partial f_{a}(z)}{\partial z_{l}}\right) \mid \leq \alpha \log \prod_{k=1}^{m} \frac{1+\left|z_{k}\right|}{1-\left|z_{k}\right|}
$$

Thus

$$
\left|\log \frac{\frac{\partial f}{\partial z_{l}}\left(\phi_{a}(z)\right)\left(1-\left|z_{l}\right|^{2}\right)}{\frac{\partial f}{\partial z_{l}}(a)\left(1+\overline{a_{l}} z_{l}\right)^{2}}\right| \leq \alpha \log \prod_{k=1}^{m} \frac{1+\left|z_{k}\right|}{1-\left|z_{k}\right|}
$$

which is equivalent to $(3.1)$.

$2^{0}$. Now, let a holomorphic function $g$ satisfies $(3.1)$ (with $g(\mathbf{O})=0$ ). Let us consider a function

$$
f(z)=\int_{0}^{z_{l}} \exp g\left(z_{1}, \cdots, z_{l-1}, s, \cdots, z_{m}\right) d s .
$$

Then

$$
\left|\log \frac{\frac{\partial f}{\partial z_{l}}\left(\phi_{a}(z)\right)\left(1-\left|z_{l}\right|^{2}\right)}{\frac{\partial f}{\partial z_{l}}(a)\left(1+\overline{a_{l}} z_{l}\right)^{2}}\right| \leq \alpha \log \prod_{k=1}^{m} \frac{1+\left|z_{k}\right|}{1-\left|z_{k}\right|}
$$

Thus

$$
\mid \Re\left[\log \frac{\partial f}{\partial z_{l}}\left(\phi_{a}(z)\right)-\log \frac{\partial f}{\partial z_{l}}(a)-2 \log \left(1+\overline{a_{l}} z_{l}\right)\right] \leq \alpha \log \prod_{k=1}^{m} \frac{1+\left|z_{k}\right|}{1-\left|z_{k}\right|}-\log \left(1-\left|z_{l}\right|^{2}\right) .
$$

From the above inequality (differentiating with respect to $\left|z_{k}\right|$ in $\mathbf{O}$ ) for every $k$ and $l$ we get

$$
-2 \alpha \leq \Re\left\{\frac{\frac{\partial^{2} f(a)}{\partial z_{l} \partial z_{k}}\left(1-\left|a_{k}\right|^{2}\right) e^{i \theta_{k}}}{\frac{\partial f}{\partial z_{l}}(a)}-2 \overline{a_{l}} e^{i \theta_{k}} \delta_{k}^{l}\right\} \leq 2 \alpha,
$$

Hence ord $f=\alpha, f \in U_{\alpha}^{l}$ and

$$
\max _{k=1, \cdots, m} \sup _{a \in \Delta^{m}}\left|\frac{\partial g}{\partial z_{k}}(a)\right|\left(1-\left|a_{k}\right|^{2}\right) \leq 2(\alpha+1) .
$$

Thus $g \in B$.

Now, we give corollaries.

COROLlary 3.1. The condition (3.1) we give in the following equivalent form:

$$
\left|g\left(\phi_{a}(z)\right)-g(a)\right| \leq \frac{K_{g}}{2} \log \prod_{k=1}^{m} \frac{1+\left|z_{k}\right|}{1-\left|z_{k}\right|},
$$

where the best constant $K_{g}$ is equal $\|g(z)-g(\mathbf{O})\|_{B}$. 
Proof. If $g \in B$, then

$$
\begin{gathered}
\left|g(z)-g\left(z_{l}^{\prime}\right)\right|=\left|\int_{0}^{z_{l}} \frac{\partial g}{\partial z_{l}}\left(z_{1}, \cdots, z_{l-1}, \cdots, z_{l}\right) d s\right| \leq \\
\int_{0}^{\left|z_{l}\right|} \frac{K_{g}}{1-r^{2}} d r=\frac{K_{g}}{2} \log \frac{1+\left|z_{l}\right|}{1-\left|z_{l}\right|},
\end{gathered}
$$

where $z_{l}^{\prime}=\left(z_{1}, \cdots, z_{l-1}, 0, \cdots, z_{m}\right)$. Using this scheme we get

$$
|g(z)-g(\mathbf{O})| \leq \frac{K_{g}}{2} \log \prod_{k=1}^{m} \frac{1+\left|z_{k}\right|}{1-\left|z_{k}\right|} .
$$

If $g \in B$, then $g\left(\phi_{a}(z)\right)-g(a) \in B$. Thus, by (3.2) we get the result.

Now, let (3.1') be fulfilled. Then for all $a \in \Delta^{m}$ :

$$
\left|\Re\left\{g\left(\phi_{a}(z)\right)-g(a)\right\}\right| \leq \frac{K_{g}}{2} \log \prod_{k=1}^{m} \frac{1+\left|z_{k}\right|}{1-\left|z_{k}\right|} .
$$

Differentiating with respect to $z_{k}=\left|z_{k}\right| e^{i \theta_{k}}$ in a neighbourhood of $\mathbf{O}$ we get

$$
\mid \Re\left\{\frac{\partial g}{\partial z_{k}}(a)\left(1-\left|a_{k}\right|^{2}\right) e^{i \theta_{k}}\right\} \leq K_{g},
$$

for all $k=1, \cdots, m$. Thus

$$
\frac{\partial g}{\partial z_{k}}(a) \mid\left(1-\left|a_{k}\right|^{2}\right) \leq K_{g}
$$

for all $a \in \Delta^{m}$ and this ends the proof.

COROLLARY 3.2. The following conditions are equivalent:

(i) $g \in B$

(ii) the family of functions $g\left(\phi_{a}(z)\right)-g(a)$ is finitely normal for $a \in \Delta^{m}$.

P r o of. Let $g \in B$. By (3.1) we get that $g\left(\phi_{a}(z)\right)-g(a)$ belongs to the class $B$ for all $a \in \Delta^{m}$. Thus we have (ii).

Now, let (ii) is fulfilled. The for every sequence $a_{n} \in \Delta^{m}$ there exists a subsequence $a_{n_{p}}$ such that a sequence $G_{p}(z)=g\left(\phi_{a_{n_{p}}}(z)-g\left(a_{n_{p}}\right)\right.$ is uniformly convergent, in $\Delta^{m}$ to an analytic function (which is not equal $\infty$ ). For every $k$ the function $\frac{\partial}{\partial z_{k}} G_{p}(z)$ is uniformly convergent to an analytic function. Thus for every $k$ the function $\frac{\partial}{\partial z_{k}} g\left(\phi_{a}(z)\right)$ is uniformly bounded (with respect to $a \in \Delta^{m}$ ) on compact sets $K \subset \Delta^{m}$. Then there is a constant $K_{g}$ such that

Thus

$$
\left|\frac{\partial}{\partial z_{k}} g\left(\phi_{a}(z)\right)\right|=\left|\frac{\partial g}{\partial z_{k}}\left(\phi_{a}(z)\right) \frac{1-\left|a_{k}\right|^{2}}{\left(1+\overline{a_{k}} z_{k}\right)^{2}}\right| \leq K_{g} .
$$

for all $a \in \Delta^{m}$.

$$
\left|\frac{\partial g}{\partial z_{k}}(a)\left(1-\left|a_{k}\right|^{2}\right)\right| \leq K_{g}
$$

Corollary 3.3. Let $g \in B, \lambda \in[0,2 \pi]$. Then the function $g(z)-g(\mathbf{O})$ maps the polydisc $\{z: z \in \mathbb{C},|z| \leq r\}^{m}$ into a domain with the boundary:

$$
\alpha e^{i \lambda}\left(\log \left(\frac{1+r}{1-r}\right)^{m-1}+2 \Xi\left(r, \frac{\sin \lambda}{\alpha}\right)\right)-\log \left(1-|r|^{2}\right) .
$$


4. Class $\dot{U}_{\alpha}^{l}$. Now, we define a class of derivatives of functions from the class $U_{\alpha}^{l}$ and give some of its properties.

Definition 4.1. $\dot{U}_{\alpha}^{l}=\left\{\frac{\partial f}{\partial z_{l}}: f \in U_{\alpha}^{l}\right\}$.

The following result give a relationship between classes $U_{\alpha}^{\prime}(l)$ (thus between classes $\left.U_{\alpha}^{l}\right)$ with different parameters $l$.

TheOREM 4.1. For all $\alpha \geq 2$ and all $l, k, n \in\{1, \cdots, m\}$ :

$$
\dot{U}_{\alpha-1}^{n} \subset \dot{U}_{\alpha}^{l} \subset \dot{U}_{\alpha+1}^{k} .
$$

Proof. From the Definitions of $U_{\alpha}^{l}$ and $U_{\alpha}^{\prime}(l)$ it follows that $h \in \dot{U}_{\alpha}^{l}$ if and only if

(i) $h(\mathbf{O})=1, h(z) \neq 0$ for $z \in \Delta^{m}$,

(ii) for all $a \in \Delta^{m}$ and $t=\left[t_{1}, \cdots, t_{m}\right] \in \mathbb{R}^{m}$, if $h \in \dot{U}_{\alpha}^{l}$, then

$$
\frac{h\left(\phi_{a}(z)\right)}{h(a)\left(1+\overline{a_{l}} z\right)^{2}} \in \dot{U}_{\alpha}^{l}
$$

and

$$
h\left(z e^{i t}\right) \in \dot{U}_{\alpha}^{l}, \quad \text { where } \quad z e^{i t}=\left[z_{1} e^{i t_{1}}, \cdots, z_{m} e^{i t_{m}}\right],
$$

(iii) if $h(z)=1+c_{1} z_{1}+\cdots+c_{m} z_{m}+o(\|z\|)$, then

$$
\left\|\left(c_{1}, \cdots, c_{m}\right)\right\| \leq 2 \alpha .
$$

Let us suppose that $h \in U_{\alpha}^{\prime}(l)$. Let $c_{j}(a)$ be a coefficient with $z_{j}$ in the expansion of the function $\frac{h\left(\phi_{a}(z)\right)}{h(a)}$. Since $\left(1+\overline{a_{l}} z_{l}\right)^{-2}=1-2 \overline{a_{l}} z_{l}+3\left(\overline{a_{l}} z_{l}\right)^{2}+\cdots$, then

$$
\frac{h\left(\phi_{a}(z)\right)}{h(a)\left(1+\overline{a_{l}} z_{l}\right)^{2}}=1+c_{1}(a) z_{1}+\cdots+\left(c_{l}(a)-2 \overline{a_{l}}\right) z_{l}+\cdots+c_{m}(a) z_{m}+o(\|z\|) .
$$

Using (iii) we get

$$
\left\|\left(c_{1}(a), \cdots, c_{l}(a)-2 \overline{a_{l}}, \cdots, c_{m}(a)\right)\right\| \leq 2 \alpha
$$

for all $a \in \Delta^{m}$. This is a necessary and sufficient condition that a function $h$ holomorphic in $\Delta^{m}$ with $h(\mathbf{O})=1$ belongs to $U_{\alpha}^{\prime}(l)$. Thus, for $k \neq l$ and all $a \in \Delta^{m}$

$$
\left\|\left(c_{1}(a), \cdots, c_{k}(a)-2 \overline{a_{k}}, \cdots, c_{m}(a)\right)\right\| \leq 2(\alpha+1),
$$

and this means that $h \in U_{\alpha+1}(k)$ and moreover $\dot{U}_{\alpha}^{l} \subset \dot{U}_{\alpha+1}^{k}$. Let us observe that for $\alpha \geq 2$ we have $\dot{U}_{\alpha+1}^{n} \subset \dot{U}_{\alpha}^{l}$.

Remark 4.1. Let us consider the function

$$
h_{o}(z)=\frac{1}{\left(1-z_{k}\right)^{2}}=\frac{\partial}{\partial z_{k}} \frac{z_{k}}{1-z_{k}} \in \dot{U}_{1}^{k} .
$$

For the function $h_{o}$ we have that $c_{k}(a)=\frac{2\left(1-\left|a_{k}\right|^{2}\right)}{1-a_{k}}$, and $\sup _{a \in \Delta^{m}}\left|c_{k}(a)\right|=4$. Thus $h_{o} \notin U_{\alpha}^{l}$ for $\alpha<2$. Moreover, we see that in Theorem 4.1 the constant $\alpha+1$ is the best.

Theorem 4.2. For all $\alpha \in<1, \infty)$ the family $\dot{U}_{\alpha}^{l}$ is compact in the topology inducted by locally uniform convergence in $\Delta^{m}$. 
Proof. From Theorems 1.1 and 2.1 it follows that for every compact set $K \subset \Delta^{m}$ and every function $h \in \dot{U}_{\alpha}^{l}$ there exists a constant $C(\alpha, K)$ such that

$$
\sup _{z \in K} \frac{\|\nabla h(z)\|}{1+|h(z)|^{2}} \leq C(\alpha, K) .
$$

From the result of R.M. Timoney ([4], theorem 3.10) it follows that $\dot{U}_{\alpha}^{l}$ is a normal family.

If $h_{n} \in \dot{U}_{\alpha}^{l}$ is a sequence which converges uniformly to $h$ on a compact $K \subset \Delta^{m}$, then, by the Theorem 2.1, $h(\mathbf{O})=1$ and $h(z) \neq 0$ in $\Delta^{m}$. Thus $h$ is in $\dot{U}_{\alpha}^{l}$, if the conditions (ii), (iii) in the proof of Theorem 4.1 are fulfield. But these conditions are equivalent to the following one

$$
\operatorname{ord}_{l} \int_{0}^{z_{l}} h(s) d s_{l} \leq \alpha,
$$

where $s=\left(s_{1}, \cdots, s_{m}\right)$; which is equivalent to

$$
\max _{k=1, \cdots, m} \sup _{z \in \Delta^{m}}\left|\frac{\frac{\partial h}{\partial z_{l}}(z)}{h(z)} \frac{1-\left|z_{k}\right|^{2}}{2}-\overline{z_{k}} \delta_{k}^{l}\right| \leq \alpha .
$$

The above inequality is true for $h_{n}$ and thus for $h$, too.

Remark 4.2. Let us observe that unlike the case $m=1$, the families $U_{\alpha}^{l}$ are not compact in the topology inducted by locally uniform convergence in $\Delta^{m}$, for $m \geq 2$.

Indeed, let

$$
Q\left(z^{l}\right)=Q\left(z_{1}, \cdots, z_{l-1}, z_{l+1}, \cdots, z_{m}\right)
$$

be a function holomorphic in $\Delta^{m-1}$ such that $Q(\mathbf{O})=0$. Then for a fixed function $h \in \dot{U}_{\alpha}^{l}$ we have

$$
f(z)=\int_{0}^{z_{l}} h(s) d s_{l}+Q\left(z^{l}\right) \in U_{\alpha}^{l} .
$$

Now, we can take a sequence of functions $Q_{n}\left(z^{l}\right)$ (of the type of the function $Q\left(z^{l}\right)$ ) such that for all $z \in \Delta^{m-1} \backslash\{\mathbf{O}\}, Q_{n}\left(z^{l}\right) \longrightarrow \infty$, if $n \rightarrow \infty$. But, it is not possible to choose (from the corresponding sequence $f_{n}$ ) a subsequence convergent to a function from $U_{\alpha}^{l}$.

\section{References}

[1] Ch. Pommerenke, Linear-invariante Familien analytischer Funktionen, Math. Ann., 155 (1964), 108-154.

[2] J. Godula, V. Starkov, Applications of ideas of Möbius invariance to obtaining equivalent definitions of Bloch functions, Annales Univ. Mariae Curia-Skłodowska 49 (1996), 41-58.

[3] —, Equivalent definitions of universal linearly-invariant familie, Materiały XI Konferencji Szkoleniowej z Teorii Zagadnień Ekstremalnych, Łódź, 1990, 34-38.

[4] R.M. Timoney, Bloch functions in several complex variables, I, Bull. London Math. Soc., 12 (1980), 241-267. 